Check for updates

Cite this: RSC Adv., 2017, 7, 53057

Received 25th September 2017

Accepted 1st November 2017

DOI: 10.1039/c7ra10611d

rsc.li/rsc-advances

\section{Chiral carbon dots and their effect on the optical properties of photosensitizers $\dagger$}

\author{
Manash Jyoti Deka and Devasish Chowdhury (D)* \\ In this work, we have successfully prepared chiral carbon dots from chiral precursors. We prepared a few \\ D-carbon dots using D-methionine, D-glucose, D-glucosamine, and L-carbon dots from L-methionine, \\ L-aspartic acid and L-alanine. We also demonstrate that the chirality of the carbon dots can affect the \\ optical properties of photosensitizer molecules like azobenzene. In the presence of azobenzene, \\ D-carbon dots and L-carbon dots interacted differently when irradiated with UV light, as azobenzene in \\ the presence of UV transforms from stable trans to metastable cis form. Thus azobenzene can react \\ differently with D- and L-carbon dots when irradiated with UV light.
}

\section{Introduction}

Carbon dots, ${ }^{1}$ the new fluorophore member of the carbon hybrid nanomaterials family, have recently attracted the attention of the scientific community and researchers worldwide. Carbon dots have a size less than $10 \mathrm{~nm}$, consist of mainly $\mathrm{sp}^{3}$ hybridized carbon unlike graphene ${ }^{2,3}$ and GQDs, ${ }^{4}$ and show bright ${ }^{5}$ fluorescence. The main advantages of carbon dots are that they possess good photoluminescence properties, chemical stability, and biocompatibility, are easy to prepare, have low cytotoxicity, are environmentally friendly, etc. ${ }^{6}$ Due to these exceptional properties, carbon quantum dots have distinguishable differences from other metal quantum dot systems. Carbon dots have potential applicability in various fields like chemistry, biology or medicine as PL sensors, ${ }^{7}$ in bioimaging, ${ }^{8}$ in drug delivery vehicles, ${ }^{9}$ in catalysis, ${ }^{10}$ and in various optoelectronic ${ }^{11}$ devices. Until now, various strategies for the synthesis of carbon dots have been explored, like hydrothermal, microwave assisted, electrochemical carbonization, chemical ablation, etc. $^{12}$

The synthesis of chiroptical ${ }^{13}$ nanoparticles is a great challenge nowadays as the conformation and molecular reorganization play a vital role in various fields, such as negative refractive materials, ${ }^{14}$ chiro plasmonic high-sensitivity bioanalysis, ${ }^{15}$ stereoselective reactions, chiral memory applications, ${ }^{16}$ hyperbolic meta-surfaces, ${ }^{17}$ chiral catalysis,${ }^{18}$ chiral sensors, ${ }^{19}$ etc. There have been only a few reports on the synthesis of chiral quantum dots. The generation of chirality on

Material Nanochemistry Laboratory, Physical Sciences Division, Institute of Advanced Study in Science and Technology, Paschim Boragaon, Garchuk, Guwahati-781035, India.E-mail: devasish@iasst.gov.in

$\dagger$ Electronic supplementary information (ESI) available: UV-visible spectra, powder XRD, FTIR and Raman spectra of D-methionine, D-glucose, D-glucosamine, L-methionine, L-aspartic acid and L-alanine. SEM images and DLS data of carbon dots synthesized from chiral precursors. Zeta potential and specific rotation of $\mathrm{D}$ and $\mathrm{L}$ carbon dots. See DOI: 10.1039/c7ra10611d graphene quantum dots (GQDs) by covalent attachment of $\mathrm{L} / \mathrm{D}^{-}$ cysteine moieties to the edges was first reported by N. Suzuki et $a .^{20} \mathrm{~J}$. K. Choi and his co-workers first demonstrated that the chirality of quantum dots could be inverted and tuned, without changing the absolute configuration of the ligand but by only modifying the structure of the chiral capping ligands. ${ }^{21} \mathrm{M}$. V. Mukhina et al. have demonstrated a new route of enantiomeric biomolecular recognition using chiral CdSe quantum dots assays. ${ }^{22} \mathrm{M}$. V. Nakagawa and his coworkers synthesized chiral graphene quantum dots using a simple esterification reaction and they successfully transferred the chirality to pyrene molecules. ${ }^{23}$ Recently, Yalin Zhang et al. synthesized chiral carbon dots by the hydrothermal treatment of citric acid and simultaneously capping with $\mathrm{L}$ and D-cysteine to introduce chirality in the system. ${ }^{24}$ But in all these reports the chirality of the nanomaterials is introduced mainly by capping or by covalent modification with other chiral moieties. It is a new challenge to synthesize chiral carbon dots without functionalization, doping or capping with other chiral molecules. The main advantage of our synthesized carbon dots is that they are intrinsically chiral and need no capping or doping with other chiral moieties to induce chirality.

In this work, we have for the first time successfully synthesized fluorescent chiral carbon dots (CDs) from different chiral precursor molecules by incomplete carbonization ${ }^{25}$ of precursor molecules without attaching, doping, capping or covalently modifying with other moieties. The chiral CDs were prepared from D-methionine, L-methionine, D-glucose, D-glucosamine, $\mathrm{L}$-aspartic acid and $\mathrm{L}$-alanine and are designated here as D-CDMeth, L-CD-Meth, D-CD-Glc, D-CD-Glcamn, L-CD-Asp and L-CDAla, respectively. Furthermore, it was observed that $\mathrm{D}^{-}$and L-carbon dots interacted differently with azobenzene-type photoswitchable molecules. The azobenzene molecule is regarded as the ideal photosensitizer, because when irradiated with UV light it transforms from stable trans to metastable cis form. ${ }^{26}$ 
Thus we show that azobenzene reacts differently with D- and L-CDs prepared from the same or different precursor molecules.

\section{Experimental section}

\section{Chemicals used}

Azobenzene $\left(\mathrm{C}_{12} \mathrm{H}_{10} \mathrm{~N}_{2}\right)$, D-methionine $\left(\mathrm{C}_{5} \mathrm{H}_{11} \mathrm{NO}_{2} \mathrm{~S}\right)$, L-methionine $\left(\mathrm{C}_{5} \mathrm{H}_{11} \mathrm{NO}_{2} \mathrm{~S}\right)$, D-glucose $\left(\mathrm{C}_{6} \mathrm{H}_{12} \mathrm{O}_{6}\right)$, and D-glucosamine hydrochloride $\left(\mathrm{C}_{6} \mathrm{H}_{13} \mathrm{NO}_{5} \cdot \mathrm{HCl}\right)$ were purchased from SigmaAldrich. L-aspartic acid $\left(\mathrm{C}_{4} \mathrm{H}_{7} \mathrm{NO}_{4}\right)$ and L-alanine $\left(\mathrm{C}_{3} \mathrm{H}_{7} \mathrm{NO}_{2}\right)$ were purchased from Merck, India. All the chemicals were used as obtained without further purification. The water used throughout the experiments was from a Milli-Q water purification system.

\section{Synthesis of fluorescent carbon dots (CDs) from $D-$ methionine, $\mathrm{L}$-methionine, D-glucose, $\mathrm{D}$-glucosamine, $\mathrm{L}$ - aspartic acid and $\mathrm{L}$-alanine}

For the synthesis of fluorescent chiral carbon dots (CDs), we employed a simple pyrolysis procedure for all the chiral precursor molecules. Briefly, $1 \mathrm{~g}$ of the precursor molecule is put into a glass beaker and heated to just above its melting point for 10 minutes. On heating, solid precursors are gradually changed to liquid, and the color turns from a colorless liquid to orange, implying the incomplete carbonization of the precursor molecules. These orange colored liquids are transferred dropwise to $30 \mathrm{ml}$ of sodium hydroxide solution $\left(10 \mathrm{mg} \mathrm{ml}^{-1}\right)$ under vigorous stirring for 5 minutes. Then the dispersion is ultrasonicated for 10 minutes and centrifuged at $5000 \mathrm{rpm}$ for $5 \mathrm{~min}$. Then the supernatant part of the dispersion is collected, which contains the carbon dots.

Thus we have successfully prepared a good aqueous dispersion of fluorescent chiral CDs. This dispersion of CDs is used for further characterization.

\section{Characterization}

The characterization of the synthesized CDs was carried out with a UV-visible absorption spectrophotometer (Shimadzu, UV-2600), Fourier Transform Infrared Spectrophotometer (Nicolet 6700), Transmission Electron Microscope (JEM 2100), and a Scanning Electron Microscope (Carl Zeiss $\sum$ sigma-VP). The particle size and zeta potential measurements were performed using Dynamic Light Scattering (Malvern Zetasizer Nano series, Nano ZS90). Raman analysis was performed using a Laser Micro-Raman system, Horiba Jobin Vyon, Model LabRam HR, using a $514 \mathrm{~nm}$ laser at room temperature. Fluorescence spectra were recorded on a Jasco spectrofluorometer FP-8300. An Optics Technology UV lamp (365 nm) was used to check the fluorescence intensity of the prepared chiral CDs. A Powder XRD spectrum of the CDs was recorded on a Bruker D8 Advance diffractometer. Circular Dichroism (CD) spectra were recorded using a JASCO J$815 \mathrm{CD}$ spectrometer by using a quartz cuvette with a path length of $1 \mathrm{~mm}$ and measurements were done in water. The optical rotation values of the carbon dots were measured by using a Rudolph Research Analytical Auto Pol II Automatic Polarimeter (wavelength $589 \mathrm{~nm}$ and cell length $50 \mathrm{~mm}$ ).

\section{Results and discussion}

The chirality of the carbon dots is mainly generated from the chiral precursor molecules, and the chirality is retained at the quantum level, i.e., the $\mathrm{D}$-form gives $\mathrm{D}$-carbon dots, and the $\mathrm{L}$ form gives L-carbon dots, which is schematically demonstrated in Scheme 1.

The synthesized chiral carbon dots are characterized using UV-visible spectroscopy. It is evident from Fig. 1A that all CDs show a prominent peak between 270 and $280 \mathrm{~nm}$. These peaks are due to the $\mathrm{n}-\pi^{*}$ transition of $\mathrm{OH}, \mathrm{NH}_{2}$, and carboxylic acid $(\mathrm{COOH})$ groups present on the surfaces of the CDs. Again there is no prominent difference in the UV-visible spectra of the synthesized CDs and precursors. The UV-visible spectra of all the precursor molecules are depicted in Fig. ESI1. $\dagger$ We have also investigated the crystallinity of the prepared CDs using XRD analysis. From Fig. 1B it is clear that all the CDs show a broad band between $2 \theta=20^{\circ}$ and $30^{\circ}$, which confirms the amorphous nature of all the prepared CDs. ${ }^{27} \mathrm{It}$ is interesting to note that the precursors of all the synthesized CDs are crystalline, and this is confirmed from the powder XRD data (Fig. ESI $2 \dagger$ ).

The chemical structure of the prepared fluorescent chiral CDs is also characterized with FT-IR in Fig. 2. For D-CD-Glc, peaks appeared at $3429 \mathrm{~cm}^{-1}$ for the $\mathrm{OH}$ stretching vibration and at $2832 \mathrm{~cm}^{-1}$ for the $\mathrm{C}-\mathrm{H}$ vibration, and the various peaks between $1530-1050 \mathrm{~cm}^{-1}$ are due to mixed vibrations of various groups of atoms, including the hydrogen atom, $\mathrm{HCH}, \mathrm{OCH}$, $\mathrm{COH}$, and $\mathrm{CCH}$ groups. ${ }^{28}$ From Fig. $2 \mathrm{~B}$ and $\mathrm{C}$, it can also be confirmed that D-CD-Meth and L-CD-Meth are chemically similar. The prominent peaks of D-CD-Meth and L-CD-Meth are observed at $3021 \mathrm{~cm}^{-1}, 2841 \mathrm{~cm}^{-1}, 1602 \mathrm{~cm}^{-1}, 1400 \mathrm{~cm}^{-1}$, $1200 \mathrm{~cm}^{-1}$ and $750-600 \mathrm{~cm}^{-1}$. The peak at $3021 \mathrm{~cm}^{-1}$ is due to the $\mathrm{N}-\mathrm{H}$ stretching bond, $2841 \mathrm{~cm}^{-1}$ is due to the $\mathrm{C}-\mathrm{H}$ stretching vibration, and the $1602 \mathrm{~cm}^{-1}$ peak is due to the $\mathrm{C}=\mathrm{O}$ stretching vibration. The peaks between $1400 \mathrm{~cm}^{-1}$ and $1200 \mathrm{~cm}^{-1}$ are due to the OH-in plane deformation, and the peaks from $750 \mathrm{~cm}^{-1}$ to $600 \mathrm{~cm}^{-1}$ are due to C-S-C asymmetric stretching and C-S symmetric stretching vibration. ${ }^{29}$

In Fig. 2D for $\mathrm{L}-\mathrm{CD}-\mathrm{Asp}$, the prominent peaks arose at $3026 \mathrm{~cm}^{-1}, 2807 \mathrm{~cm}^{-1}, 1696 \mathrm{~cm}^{-1}, 1610 \mathrm{~cm}^{-1}, 1310 \mathrm{~cm}^{-1}$ and

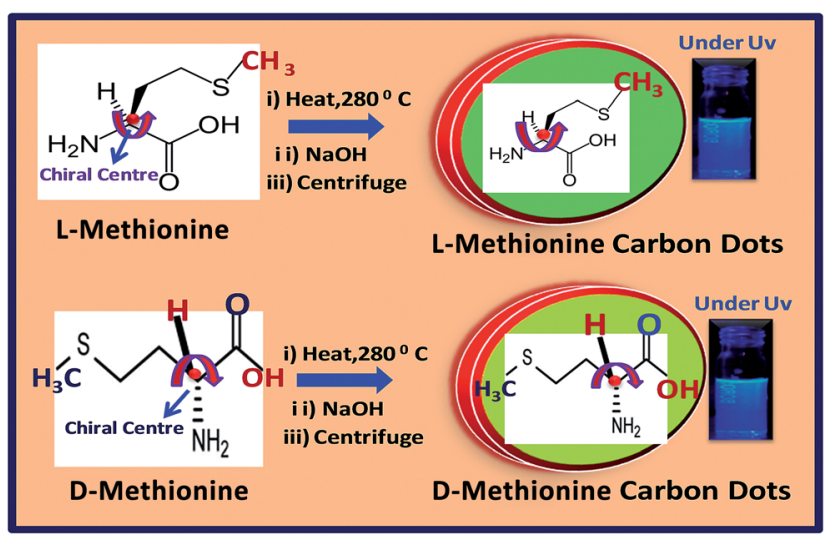

Scheme 1 Schematic representation of the synthesis of $D$ - and $\mathrm{L}$-carbon dots from $\mathrm{D}$ - and L-methionine precursor molecules. 

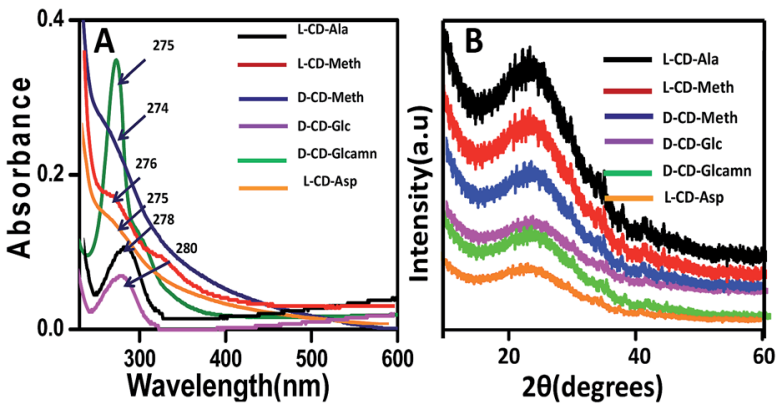

Fig. 1 (A) UV-visible spectra and (B) XRD spectra of L-CD-Ala, L-CDMeth, D-CD-Meth, D-CD-Glc, D-CD-Glcamn, and L-CD-Asp.

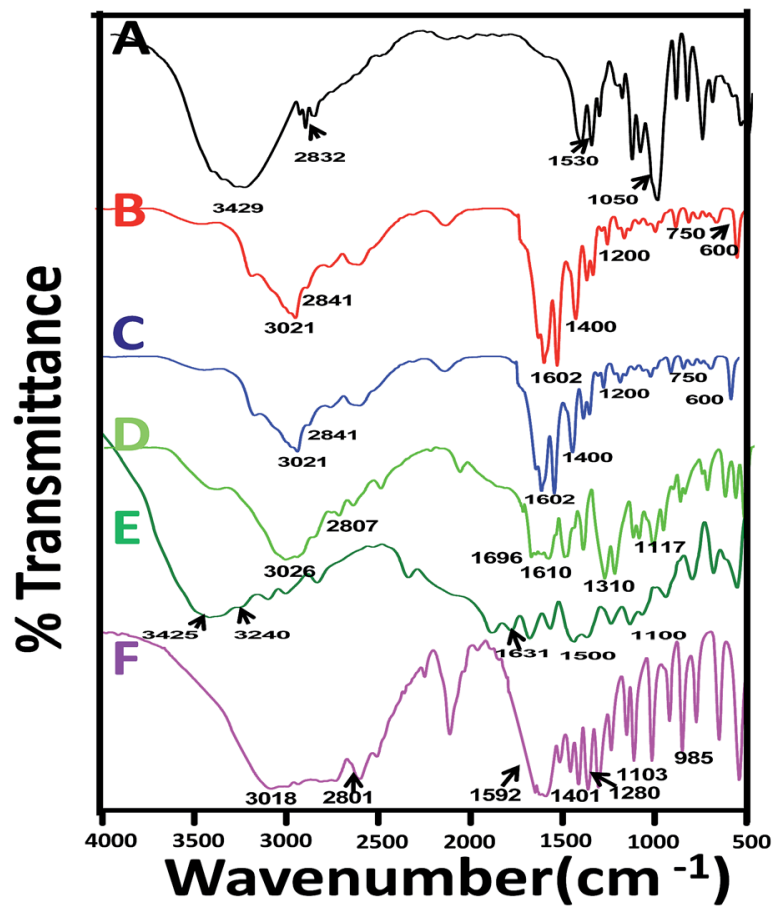

Fig. 2 FT-IR spectra of (A) D-CD-Glc, (B) D-CD-Meth, (C) L-CD-Meth, (D) L-CD-Asp, (E) D-CD-Glcamn, (F) L-CD-Ala.

$1117 \mathrm{~cm}^{-1}$. We assigned the peaks at $3026 \mathrm{~cm}^{-1}$ to $\mathrm{NH}$ symmetric stretching; the $2807 \mathrm{~cm}^{-1}$ peak is due to $\mathrm{CH}$ stretching in $\mathrm{CH}_{2}$; the peak at $1696 \mathrm{~cm}^{-1}$ is due to $\mathrm{C}=\mathrm{O}$ symmetric stretching; $1610 \mathrm{~cm}^{-1}$ is due to $\mathrm{C}=\mathrm{O}$ asymmetric stretching; $1310 \mathrm{~cm}^{-1}$ is due to $\mathrm{CH}$ bending and a peak at $1117 \mathrm{~cm}^{-1}$ is due to $\mathrm{C}-\mathrm{OH}$ symmetric stretching in $\mathrm{COOH} .{ }^{30}$ The main peaks for D-CD-Glcamn are observed at $3426 \mathrm{~cm}^{-1}$, $3240 \mathrm{~cm}^{-1}, 2807 \mathrm{~cm}^{-1}, 1631 \mathrm{~cm}^{-1}$, and $1500-1100 \mathrm{~cm}^{-1}$ (Fig. 2E). The peaks are assigned as $3426 \mathrm{~cm}^{-1}$ due to $\mathrm{OH}$ stretching vibration, $3240 \mathrm{~cm}^{-1}$ due to $\mathrm{N}-\mathrm{H}$ stretching vibration, and $1631 \mathrm{~cm}^{-1}$ due to $\mathrm{N}-\mathrm{H}$ bending vibration. All the prominent peaks between $1100 \mathrm{~cm}^{-1}$ and $1500 \mathrm{~cm}^{-1}$ are for mixed vibrations of $\mathrm{HCH}, \mathrm{COH}, \mathrm{CCH}$, and $\mathrm{OCH}$ groups. For L-CD-Ala (Fig. 2F) the characteristic peaks are observed at $3018 \mathrm{~cm}^{-1}$ (asymmetric $\mathrm{NH}_{2}$ stretching), $2801 \mathrm{~cm}^{-1}$ (asymmetric $\mathrm{CH}$ stretching of $\left.\mathrm{CH}_{3}\right)$, and $1592 \mathrm{~cm}^{-1}\left(\mathrm{NH}_{2}\right.$ bending vibration). The peaks at $1401 \mathrm{~cm}^{-1}, 1280 \mathrm{~cm}^{-1}, 1103 \mathrm{~cm}^{-1}$ and $985 \mathrm{~cm}^{-1}$ are due to $\mathrm{NH}_{2}$ bending, $\mathrm{NH}_{2}$ scissoring, $\mathrm{CH}_{3}$ puckering and $\mathrm{H}-\mathrm{N}-\mathrm{C}-\mathrm{C}$ band vibration. ${ }^{31}$ We have also analyzed the FT-IR spectra of the precursor molecules and they are depicted in Fig. ESI3. $\uparrow$ The peaks for the precursor molecules are sharper and more intense compared to those for the CDs.

This can be attributed to the restriction of the vibrations due to the formation of the carbon dots. However, the range in which the peaks are positioned remains the same. The Raman spectra for all the precursors were performed and are shown in Fig. ESI4 (ESI† Section). We are unable to perform the Raman analysis of the CDs, as the synthesised CDs are highly fluorescent which interferes in the Raman analysis.

Scanning electron microscopy images confirmed the quantum size of the prepared chiral CDs, and are depicted in Fig. 3A and B. From SEM (Fig. 3A and B) it is confirmed that the average size of the prepared CDs was below $10 \mathrm{~nm}$ and the size is uniformly distributed. We also performed the DLS technique and confirmed that the prepared chiral CDs are of a size below $10 \mathrm{~nm}$ (Fig. ESI6 $\dagger$ ). Transmission electron microscopy (TEM) image characterization was also performed for $\mathrm{D}$-CD-Meth and $\mathrm{L}$-CD-Meth to determine the size and morphology of the synthesized CDs dispersed in water (Fig. 3C and D). The images show that the size of the chiral carbon dots is below $10 \mathrm{~nm}$, uniform and with a spherical morphology. The size of the CDs ranges from 2 to $5 \mathrm{~nm}$. This is indeed in good agreement with the SEM analysis and DLS. The selected area (electron) diffraction patterns of D-CD-Meth and L-CD-Meth were also carried out (inset: Fig. 3C and D). There is no prominent diffraction phase observed, which proves that the carbon dots are amorphous and supports the XRD data.

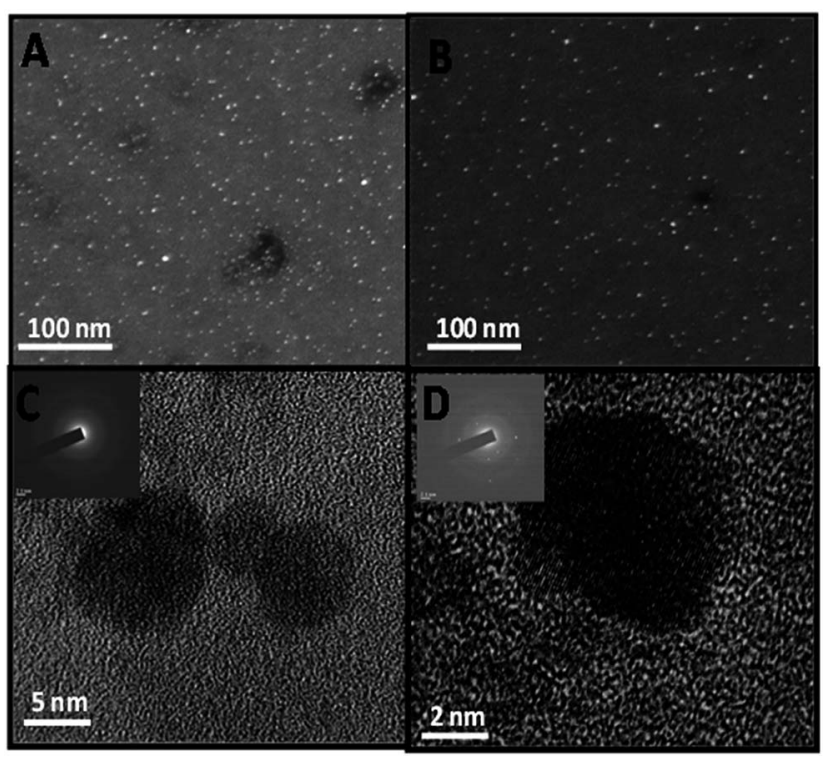

Fig. 3 Representative scanning electron microscope images of (A) DCD-Meth, (B) L-CD-Meth and the corresponding high-resolution transmission electron microscopy images of (C) D-CD-Meth, (D) L-CDMeth [inset: selected area (electron) diffraction pattern of carbon dots]. 
The surface charges of the prepared chiral CDs were also investigated using zeta potential measurement, and it was found to be negative. The negative value of the surface charge can be attributed to the presence of different functionalities $\left(-\mathrm{COOH}, \mathrm{OH}, \mathrm{NH}_{2}\right.$, etc.) on the surface of the $\mathrm{CDs}$ and it is depicted in Table ESI7 (ESI $\dagger$ ). The negative zeta means that more carboxylic groups are on the surface compared to $\mathrm{NH}_{2}$. Besides that, some $\mathrm{NH}_{2}$ groups are also present on the surface (confirmed from FT-IR spectra) which may decrease the net surface charge from more negative to less negative.

The PL properties of all the synthesized CDs were studied in detail and are depicted in Fig. 4. The stacked PL spectra obtained after excitation of the CDs at wavelengths ranging from $300 \mathrm{~nm}$ to $450 \mathrm{~nm}$ are shown in Fig. 4A-C. From Fig. $4 \mathrm{~A}-\mathrm{C}$ it is interesting to note that with an increase in the excitation wavelength from $300 \mathrm{~nm}$ to $450 \mathrm{~nm}$ for L-CD-Ala, L-CD-Meth, D-CD-Glc, first the emission from the CDs increased and shifted and showed emission maxima at a certain wavelength, then the intensity of the emission gradually decreased. As-prepared L-CD-Ala, L-CD-Meth, and D-CD-Glc show excitation-dependent PL properties, as shown by other carbon dots and GQDs reported earlier. ${ }^{32,33}$ The origin of excitation-dependent or excitation-independent PL properties has led to many hypotheses, from particle size distribution to the presence of different emissive states and solvent relaxation around CDs, and the participation of discrete multiple electronic states in the carbon dot system. ${ }^{34}$ The emission maxima for L-CD-Ala, L-CD-Meth and D-CD-GLC are at wavelengths of $320 \mathrm{~nm}, 300 \mathrm{~nm}$, and $320 \mathrm{~nm}$, respectively. But on the other hand D-CD-Meth, L-CD-Meth and L-CD-Ala show the opposite behavior, which is depicted in Fig. 4D-F. These CDs show excitation-independent PL properties, as shown by our previous report on carbon dots synthesized from paper ash. The emission maxima for D-CDMeth, L-CD-Meth and L-CD-Ala, are observed at 310, 310 and $330 \mathrm{~nm}$, respectively. Our synthesized CDs all have a size less than $10 \mathrm{~nm}$ and are dispersed in the same solvent (water), so there is no effect of size and solvents on the PL behavior of CDs. From the investigation of the PL spectra, it is confirmed that chirality also has no role in the fluorescence properties of carbon dots. The exact mechanism is not well known or understandable, but for our case all the chiral CDs mainly differ in their functional groups (the exceptions are $\mathrm{D}$ - and L-methionine carbon dots). There is a report that surface functionalities also affect the PL properties of carbon nanomaterials. ${ }^{35}$

The chiroptical activity of synthesized carbon dots is investigated through circular dichroism spectroscopy (CD). In Fig. 5, representative $\mathrm{CD}$ spectra of $\mathrm{D}^{-}$and $\mathrm{L}$-CD-Meth are demonstrated. It can be observed that in carbon dot synthesis from $\mathrm{D}^{-}$ methionine (D-Meth) the CD peak appeared at $210 \mathrm{~nm}$ and it is

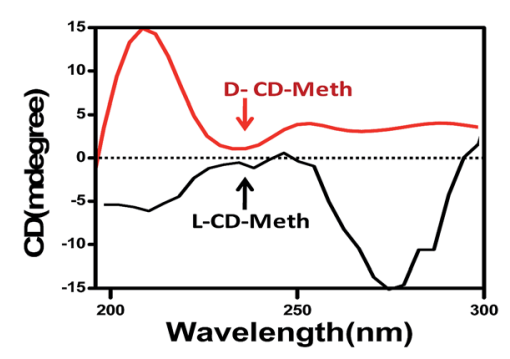

Fig. 5 Circular dichroism spectra for D-CD-Meth (red) and L-CD-Meth (black).
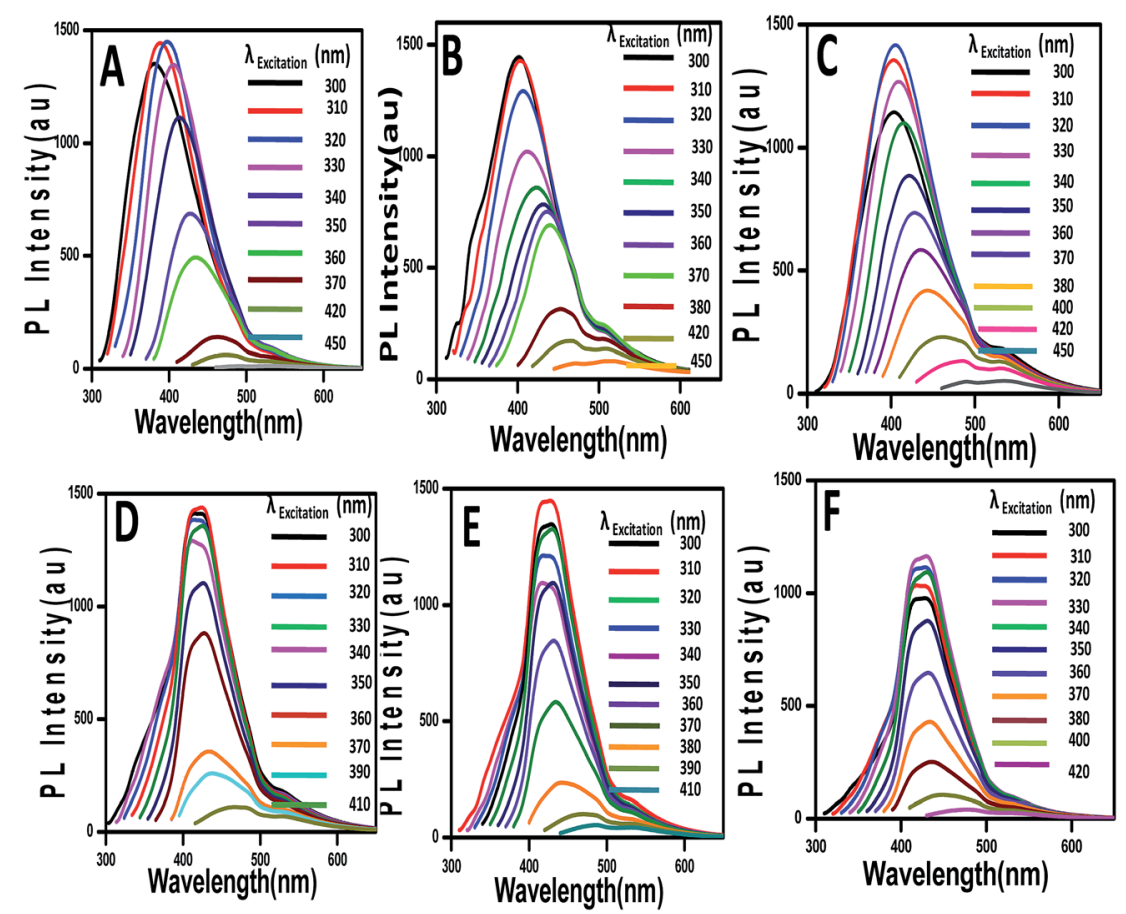

Fig. 4 Stacked PL spectra of (A) D-CD-Glcamn, (B) D-CD-GlC, (C) L-CD-Asp, (D) D-CD-Meth, (E) L-CD-Meth and (F) L-CD-Ala. 
in the positive direction (+sign). But, interestingly, carbon dots originating from L-methionine show different behavior. The peak for L-CD-Meth appears at $270 \mathrm{~nm}$, and it rotates the polarized light in the negative direction.

Thus, L-CD-Meth gives a negative CD Cotton effect, while D-CD-Meth shows a positive CD Cotton effect due to their characteristic change in circular dichroism in the vicinity of the absorption band. In D-CD and L-CDs the configurations of the groups are different so their specific rotations are also different (Table ESI $4 \dagger)$. The redshifting $(210 \mathrm{~nm}$ to $270 \mathrm{~nm}$ ) of the CD spectra for the same precursor molecules (D- and L-methionine) may be due to the absorption flattening (AF) and circular differential scattering phenomena exhibited by biomolecules. ${ }^{36}$ Castiglioni et al. showed that absorption flattening (AF) and light scattering (LS) are mainly observed in the case of an aggregate system, and this is true for our carbon dot system as we synthesize carbon dots using a bottom-up approach from different biomolecules. ${ }^{37}$ There is a report that solvents can also affect the CD spectra. ${ }^{38}$ Again the CD spectra of D- and L-Meth CDs do not show enantiomeric behavior, in spite of their synthesis from the same precursor. The reason is that because we have synthesised the CDs through hydrothermal routes (a chemical approach), this affects the enantiomeric form of the CDs. But from the CD spectra, it can be confirmed that chirality is retained in the carbon dots themselves, which show the same chiroptical activity as that of the precursors molecules, i.e., D-carbon dots arose from D-parent molecules and L-carbon dots from $\mathrm{L}$-parent molecules. This is true for the same or different precursor molecules.

The specific rotation values of carbon dots are measured by a Rudolph Research Analytical Auto Pol II Automatic Polarimeter (wavelength $589 \mathrm{~nm}$, cell length $50 \mathrm{~mm}$ and temperature $25^{\circ} \mathrm{C}$ ). The specific rotation values of carbon dots synthesised from $\mathrm{D}^{-}$and $\mathrm{L}$-precursor molecules are found to be positive and negative, respectively and are depicted in Table ESI8. $\dagger$ From the specific rotation values, it is confirmed that the chirality is also retained in carbon dots.

\section{Interaction of $\mathrm{D}^{-}$and $\mathrm{L}-\mathrm{CDS}$ with azobenzene}

It is well known that a chiral moiety shows "chiral interaction": in other words, it will interact differently with photosensitizer molecules. Thus the next objective was to study the interaction of chiral carbon dots with a photosensitizer molecule. We have chosen azobenzene as a model photosensitizer.

It was observed that azobenzene which is in the trans form does not interact with D-CDs or L-CDs. However, there is a drastic change in the UV-visible spectrum when ${ }^{-}$-CDs and $\mathrm{L}^{-}$ CDs interact with azobenzene in the presence of UV light. Azobenzene transformed from the trans isomer to the cis isomer when irradiated with UV light (shown in Fig. ESI9†). ${ }^{24}$

For UV-visible spectra measurement, we made the concentration of all CDs $5 \mu \mathrm{M}$ by dilution. We also prepared a $5 \mu \mathrm{M}$ azobenzene dispersion in water. Before adding azobenzene, we measured the UV-vis spectra of all CDs and they showed nearly identical absorption profiles (both in shape and intensity). Then we mixed azobenzene with the prepared CDs in the ratio $1: 1$, heated to $60{ }^{\circ} \mathrm{C}(1 \mathrm{~h})$ to build noncovalent interactions and took the UV-vis spectra before and after UV-irradiation $(20 \mathrm{~min}$, $365 \mathrm{~nm}$ ). The non-covalent interaction of CDs and azobenzene was confirmed from FT-IR spectra analysis. It was observed that the main characteristic peaks of the chiral CDs are only shifted after interaction with azobenzene when irradiated with UV for $20 \mathrm{~min}, 365 \mathrm{~nm}$. No new peaks appeared in the spectra, which indirectly proves that there is only noncovalent interaction. The details of the experiments are given in Fig. ESI10. $\dagger$ The UVvisible spectra are shown in Fig. 6. Fig. 6A shows the spectra of D-CDs prepared from D-glucose and azobenzene without irradiation with UV light (0 min) and under UV light (365 nm) for 20 minutes. It was observed that without UV irradiation (0 min) of azobenzene/chiral CDs there is no change in the UVvisible spectrum and the absorbance remains the same. But the UV-treated azobenzene/chiral CDs system shows a drastic reduction in the intensity of prominent peaks and an observed shifting of the peaks. In D-CD-Glc/Azo the peaks are shifted to $289 \mathrm{~nm}$ and $244 \mathrm{~nm}$. The other systems, D-CD-Glcamn/Azo and D-CD-Meth/Azo, show a similar reduction in peaks. In D-CDGlcamn/Azo the peaks are observed at $315 \mathrm{~nm}$ and $250 \mathrm{~nm}$ (Fig. 6B), and in D-CD-Meth/Azo the peaks appear at $263 \mathrm{~nm}$ and $298 \mathrm{~nm}$ (Fig. 6C). The interaction of azobenzene with L-CDs under UV radiation also shows a similar trend. L-CDs/Azo irradiated in UV (20 min) are depicted in Fig. 6D-F. In Fig. 6D the $\pi-\pi^{*}$ and $\mathrm{n}-\pi^{*}$ transition peaks are shifted to $255 \mathrm{~nm}$ and $314 \mathrm{~nm}$; for L-CD-Ala/Azo and L-CD-Asp/Azo, the peaks appear at $227 \mathrm{~nm}$ and 307, respectively. The new shifted peaks for L-CDMeth/Azo are observed at $236 \mathrm{~nm}$ and $280 \mathrm{~nm}$. Interestingly for all the cases (D- and L-CDs/Azo), the $430 \mathrm{~nm}$ ( $\mathrm{n}-\pi^{*}$ transition) peak disappeared after UV irradiation for 20 minutes. Hence from the UV-visible study, it can be observed that chiral carbon dots (D- and $\mathrm{L}^{-}$) from the same or different precursor molecules interacted differently with an azobenzene-type photosensitizer when azobenzene itself changes from the trans to cis conformation. Here, we have seen that $20 \mathrm{~min}$ is the maximum time to observe this effect; beyond this no effect is observed. We also investigated the UV response of chiral CDs for the same irradiation time (20 $\mathrm{min}, 365 \mathrm{~nm}$ ) and it was observed that there is a slight shift in UV peaks for both D- and L-CDs (Fig. ESI11 $\dagger$ ). However, the change is substantial in the presence of azobenzene/chiral CDs. We should add that we have also investigated the reversibility of azobenzene and azobenzene/ chiral CDs. After the material has been irradiated with VIS light after $365 \mathrm{~nm}$, the azobenzene is again transformed to cis from trans, but for the CDs with azobenzene it does not return to its trans form after interaction with azobenzene. After interaction with azobenzene, the system becomes irreversible.

\section{Mechanism}

We have constructed and postulated a simple model to understand the difference in interaction between azobenzene with D- and L-carbon dots in UV when azobenzene is isomerized from trans to cis conformation and this is depicted in Scheme 2. In the case of chiral carbon dots ( $\mathrm{D}^{-}$and $\mathrm{L}^{-}$), the groups attached to the chiral carbon centers are oriented differently with their 

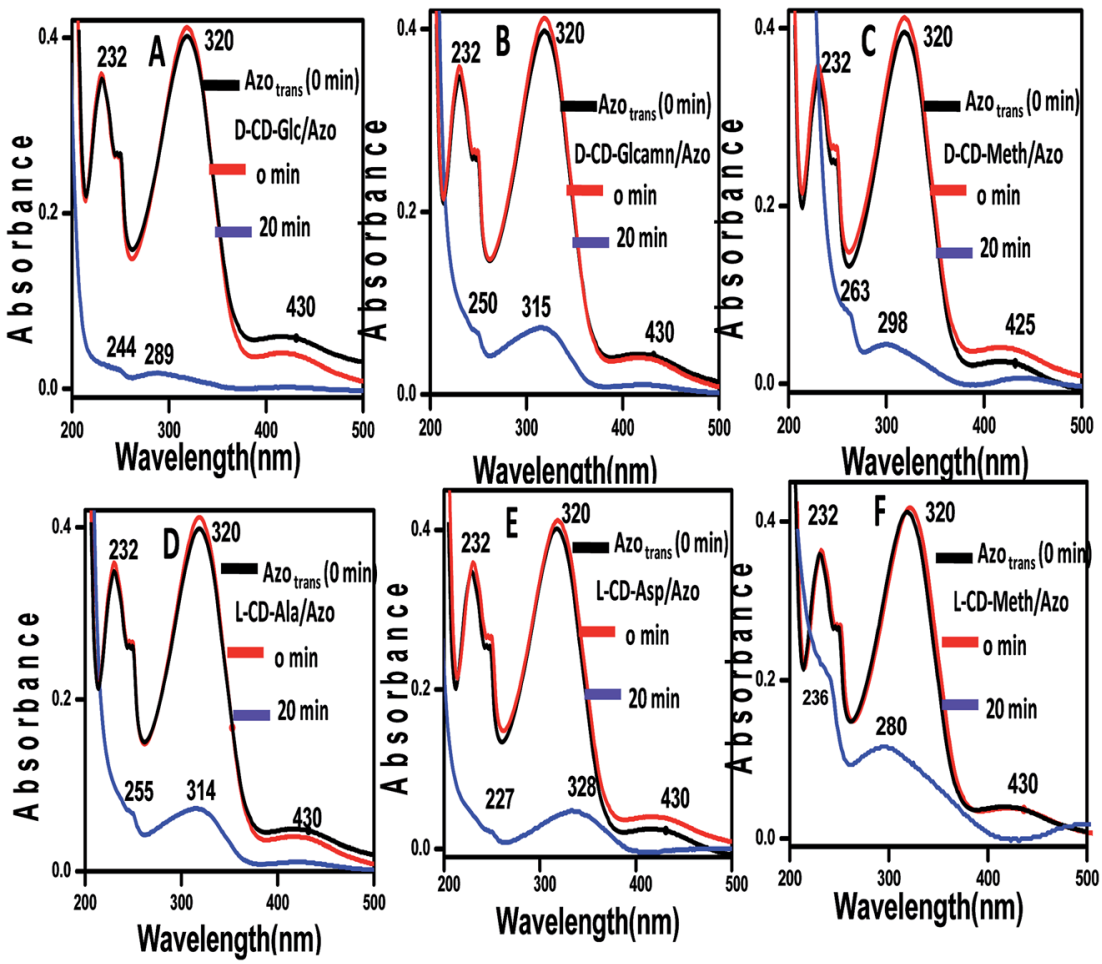

Fig. 6 UV-visible spectra of (A) D-CD-Glc/Azo, (B) D-CD-Glcamn/Azo, (C) D-CD-Meth/Azo, (D) L-CD-Ala/Azo (E) L-CD-Asp/Azo and (F) L-CDMeth/Azo for $0 \mathrm{~min}$ and $20 \mathrm{~min}$ UV treatment at $365 \mathrm{~nm}$.

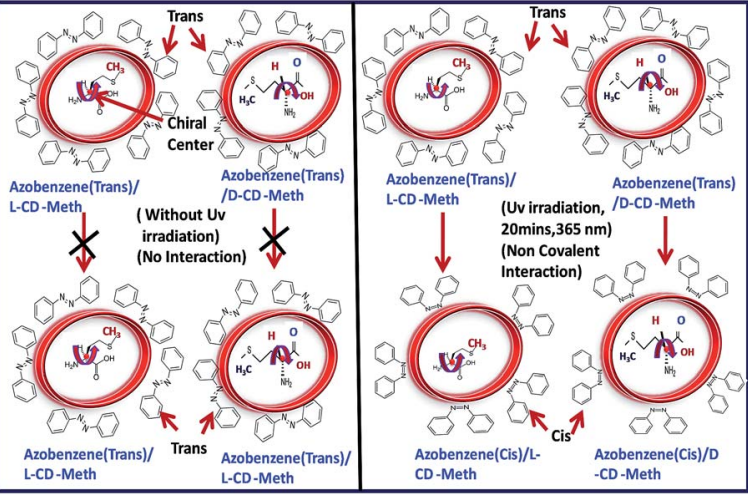

Scheme 2 Schematic representation of the interaction of azobenzene with $D$ - and L-carbon dots synthesized from D- and L-forms of the same precursor molecules (D- and L-methionine).

preferences. For example, in the case of D-CD-Meth and L-CDMeth, the groups attached to the chiral centers are the same, but their orientation is different. Again, azobenzene when irradiated with UV light (365 nm) is photoisomerized from planar trans to nonplanar cis conformation, altering their orientations. As azobenzene contains two phenyl groups, there is delocalization of $\pi$ electrons and nonbonding electrons on $-\mathrm{N}=\mathrm{N}$. These nonbinding and $\pi$-electrons can easily interact noncovalently with nonbonding electrons of $\mathrm{N}, \mathrm{S}$ and the presence of $\pi$-systems in the chiral carbon dots of $\mathrm{D}^{-}$and L-Meth. UV spectra detect these interactions well. We have observed that the change in peak intensities and shifting in UV originated from $\pi-\pi^{*}$ and $\mathrm{n}-\pi^{*}$ transitions. But as the groups attached to the $\mathrm{D}^{-}$and $\mathrm{L}^{-}$-carbon dots of the same precursors ( $\mathrm{D}^{-}$and $\mathrm{L}^{-}$) possess different orientations, so the extent of noncovalent interaction for D- and L-chiral carbon dots with azobenzenes are different for the same irradiation time (20 minutes). This may be the reason for the observed difference in intensities and shifting in UV peaks for $\mathrm{D}^{-}$and L-carbon dots which demonstrated that the interaction with azobenzene-type photosensitizer molecules is possible and different. Thus, alternatively, it is possible to identify the chirality of the carbon dots by monitoring the UV peaks interacting with azobenzene.

Scheme 2 shows that if there is no UV treatment for azobenzene/D-CD or $\mathrm{L}-\mathrm{CD}$, there is no change in UV-vis spectrum. UV treatment is needed to facilitate the interaction between azobenzene and chiral CDs because on UV treatment the azobenzene has also changed the configuration from trans to cis. But the extent of interaction of $\mathrm{D}$ - and $\mathrm{L}$-CDs is different for same irradiation time (20 min, UV $365 \mathrm{~nm}$ ) due to the configuration and orientation of the groups attached to the chiral centers being different. This is the reason why UV can detect different signals for azobenzene/D-CD or L-CD (20 min, UV $365 \mathrm{~nm})$.

\section{Conclusions}

In summary, we have successfully synthesized chiral carbon dots from chiral precursor molecules. We successfully prepared a few D-CDs D-methionine, D-glucose, D-glucosamine, and L-CDs L-methionine, $\mathrm{L}$-aspartic acid and $\mathrm{L}$-alanine. These $\mathrm{L}^{-}$and D-carbon dots interacted differently with azobenzene 
photosensitizer molecules when they transformed from the trans to the cis isomeric form. In this work, we synthesized chiral carbon dots by incomplete carbonization of precursor molecules without capping or functionalization with other chiral moieties. We think that this type of understanding of chiroptical properties of carbon nanodots will pave the way for a new direction of research on carbon hybrid nanomaterials and that it deserves further investigation.

\section{Conflicts of interest}

There are no conflicts to declare.

\section{Acknowledgements}

The authors thank SERB, New Delhi, Grant No. SB/S1/PC-69/ 2012 and BRNS, Mumbai, Grant No. 34/14/20/2014-BRNS. MJD wants to thank SERB for fellowship.

\section{References}

1 Y. Wang and A. Hu, J. Mater. Chem. C, 2014, 2, 6921.

2 M. J. Deka, U. Baruah and D. Chowdhury, Mater. Chem. Phys., 2015, 168, 236.

3 M. J. Deka and D. Chowdhury, J. Phys. Chem. C, 2016, 120, 4121.

4 X. Yan, X. Cui and L.-S. Li, J. Am. Chem. Soc., 2010, 132, 5944. 5 U. Baruah, M. J. Deka and D. Chowdhury, RSC Adv., 2014, 4, 36917.

6 H. Li, Z. Kang, Y. Liu and S.-T. Lee, J. Mater. Chem., 2012, 22, 24230.

7 U. Baruah, N. Gogoi, A. Konwar, M. J. Deka, D. Chowdhury and G. Majumdar, J. Nanopart., 2014, 2014, 178518.

8 Y. Song, S. Zhu and B. Yang, RSC Adv., 2014, 4, 27184.

9 Q. Zeng, D. Shao, X. He, Z. Ren, W. Ji, C. Shan, S. Qu, J. Li, L. Chen and Q. Li, J. Mater. Chem. B, 2016, 4, 5119.

10 Z. Zhang, T. Zheng, X. Li, J. Xu and H. Zeng, Part. Part. Syst. Charact., 2016, 33, 457.

11 X. Li, M. Rui, J. Song, Z. Shen and H. Zeng, Adv. Funct. Mater., 2015, 25, 4929.

12 P. Roy, P.-C. Chen, A. P. Periasamy, Y. N. Chen and H. T. Chang, Mater. Today, 2015, 18, 447.

13 M. Esposito, V. Tasco, F. Todisco, I. Tarantini, M. Cuscunà, L. Dominici, M. D. Giorgi and A. Passaseo, Nanoscale, 2015, 7, 18081.

14 X. Jia, Q. Meng, C. Yuan, Z. Zhou and X. Wang, Optics, 2016, 127, 5738.

15 X. Wu, L. Xu, L. Liu, W. Ma, H. Yin, H. Kuang, L. Wang, C. Xu and N. A. Kotov, J. Am. Chem. Soc., 2013, 135, 18629.
16 F. F. Milton, J. Govan, M. V. Mukhinab and Y. K. Gun'ko, Nanoscale Horiz., 2016, 1, 14.

17 S. S. Oh and O. Hess, Nano Convergence, 2015, 2, 24.

18 T. Yasukawa, H. Miyamura and S. Kobayashi, Chem. Soc. Rev., 2014, 43, 1450.

19 J. Jiao, X. Liu, X. Mao, J. Li, Y. Cheng and C. Zhu, New J. Chem., 2013, 37, 317.

20 N. Suzuki, Y. Wang, P. Elvati, Z.-B. Qu, K. Kim, S. Jiang, E. Baumeister, J. Lee, B. Yeom, J. H. Bahng, J. Lee, A. Violi and N. A. Kotov, ACS Nano, 2016, 10, 1744.

21 J. K. Choi, B. E. Haynie, U. Tohgha, L. Pap, K. Wade Elliott, B. M. Leonard, S. V. Dzyuba, K. Varga, J. Kubelka and M. Balaz, ACS Nano, 2016, 10, 3809.

22 M. V. Mukhina, I. V. Korsakov, V. G. Maslov, F. PurcellMilton, J. Govan, A. V. Baranov, A. V. Fedorov and Y. K. Gun'ko, Sci. Rep., 2016, 6, 24177.

23 M. Vazquez-Nakagawa, L. Rodrıguez-Pérez, M. A. Herranz and N. Martın, Chem. Commun., 2016, 52, 665.

24 Y. Zhang, L. Hu, Y. Sun, C. Zhu, R. Li, N. Liu, H. Huang, Y. Liu, C. Huang and Z. Kanga, RSC Adv., 2016, 6, 59956.

25 Y. Dong, J. Shao, C. C. Chen, H. Li, R. Wang, Y. Chi, X. Lin and G. Chen, Carbon, 2012, 50, 4738.

26 L.-J. Chen, J.-D. Lin and C.-R. Lee, J. Mater. Chem. C, 2014, 2, 4388.

27 J. B. Essner, C. H. Laber, S. Ravula, L. Polo-Parada and G. A. Baker, Green Chem., 2016, 18, 243.

28 D. K. Buslov, N. A. Nikonenko, N. I. Sushko and R. G. Zhbankov, J. Appl. Spectrosc., 2002, 69, 817.

29 S. Pandiarajan, M. Umadevi, M. B. Mary, R. K. Rajaram and V. Ramakrishnan, J. Raman Spectrosc., 2004, 35, 907.

30 S. Kumar, A. K. Rai, S. B. Rai, D. K. Rai, A. N. Singh and V. B. Singh, J. Mol. Struct., 2006, 791, 23.

31 M. E. Mohamed and A. M. A. Mohammed, Int. Lett. Chem., Phys. Astron., 2013, 10, 1.

32 B. Li, Y. Guo, A. Iqbal, Y. Dong, W. Li, W. Liu, W. Qin and Y. Wang, RSC Adv. , 2016, 6, 107263.

33 M. J. Deka and D. Chowdhury, ChemistrySelect, 2017, 2, 1999. 34 A. Sharma, T. Gadly, A. Gupta, A. Ballal, S. K. Ghosh and M. Kumbhakar, J. Phys. Chem. Lett., 2016, 7, 3695.

35 X. Li, S. Zhang, S. A. Kulinich, Y. Liu and H. Zeng, Sci. Rep., 2014, 4, 1-8.

36 C. L. Phillips, W. E. Mickols, M. F. Maestre and I. Tinoco Jr, Biochemistry, 1986, 25, 7803.

37 E. Castiglioni, S. Abbate, G. Longhiand and R. Gangemi, Chirality, 2007, 19, 491.

38 J. H. Bredehoft, N. C. Jones, C. Meinert, A. C. Evans, V. S. Hoffmann and U. J. Meierhenrich, Chirality, 2014, 26, 373. 